Voix et Images

volxetimages

\title{
La détresse (bibliographique) et l'enchantement (critique)
}

\section{Pierre Hébert}

Volume 18, numéro 1 (52), automne 1992

Les écritures masculines

URI : https://id.erudit.org/iderudit/201012ar

DOI : https://doi.org/10.7202/201012ar

Aller au sommaire du numéro

Éditeur(s)

Université du Québec à Montréal

ISSN

0318-9201 (imprimé)

1705-933X (numérique)

Découvrir la revue

Citer cet article

Hébert, P. (1992). La détresse (bibliographique) et l'enchantement (critique).

Voix et Images, 18(1), 191-196. https://doi.org/10.7202/201012ar d'utilisation que vous pouvez consulter en ligne.

https://apropos.erudit.org/fr/usagers/politique-dutilisation/ 


\title{
La détresse (bibliographique) et l'enchantement (critique)
}

\author{
Pierre Hébert, Université de Sherbrooke
}

\begin{abstract}
Voix et Images inaugure ici une cbronique négulière sur. les reurues. Sans être exbaustif, ce parcours tentera de dresser un repertoire d'articles variés regroupés autour de divers sujets ou approcbes. Son objet sera avant tout les études de littérature québécoise, parfois la théorie lorsqu'elle proviendra du Québec; quant à sa visée, elle sera beaucoup plus descriptive que critique.
\end{abstract}

Quel paradoxe! Pendant que nous nous donnons une mémoire littéraire impressionnante (dictionnaires, histoires littéraires, etc.), nous avons abandonné la plupart de nos entreprises relevant de la "bibliographie courante ", tant en ce qui concerne les œuvres que la critique. Sommes-nous condamnés à perpétuellement nous souvenir?

Une chronique qui propose une revue des revues aura certes toujours sa place, et plus encore dans le contexte qui vient d'être évoqué. Ce genre de parcours ne peut remplacer une bibliographie annuelle de la critique, ainsi que le faisait la feue Revue d'bistoire littéraire du Québec et du Canada français; mais cette Revue des revues peut signaler des tendances, établir des regroupements/recoupements. Il faut bien que la faconde d'une chronique offre quelques avantages sur la sobriété d'une bibliographie.

\section{Sémiotique / Théâtre}

Me sera-t-il permis, dans le cadre d'une première chronique, de signaler des numéros de revue datant de 1991, voire de 1990? C'est le cas de Horizons philosophiques, qui a marqué sa naissance avec deux numéros sous le même intitulé de : Sémiotiques ${ }^{1}$. En ce qui concerne 
la littérature, l'un des objets importants de ces deux numéros, une interrogation générale de Marc Angenot pose cette question toute simple: que faisons-nous lorsque nous étudions la littérature? Dans -Frontières des études littéraires: science de la littérature, science des discours ", Marc Angenot note avec raison que, en ce qui a trait à l'objet même des études littéraires, l'institution universitaire esquive généralement le problème. Une revue des diverses approches conduit à la conclusion :que les études littéraires ont des méthodes mais pas d'objet * (p. 30). Poursuivons tout de même au-delà de ce jugement péremptoire: -les méthodologies littéraires sont des méthodologies sociodiscursives perverties par leur application à un objet fétichisé. (p.32) ${ }^{2}$. Une ouverture de perspective s'impose pour sortir de l'ornière: «Ce qui nous manque aujourd'hui [...] c'est une théorie et une histoire du discours social . (p. 34) à laquelle travaille, on le sait, Marc Angenot. Si la solution est à venir, cet article secoue au moins cette tentation constante de la naïveté en études littéraires.

Il n'y a pas lieu, parfois du moins, de trop désespérer de la théorie: il lui arrive d'être un processus cumulatif. Après les modèles de Steen Jansen ${ }^{3}$ ou de Louis Francoeur ${ }^{4}$, Louise Vigeant propose à son tour un modèle sémiologique du théâtre, prenant en compte son objet tout à la fois comme texte et comme spectacle. Cette étude met en place les principaux niveaux d'une sémiotique du théâtre et, à cet égard, a valeur d'introduction pédagogique, chaque aspect proposé pouvant donner lieu à un développement plus détaillés. C'est d'ailleurs ce que l'auteure fait elle-même ailleurs, en l'occurrence dans un numéro de la revue Jeu consacré à la scénographie: Vigeant aborde, fidèle à son approche fonctionnaliste, les différents rôles que jouent les didascalies ${ }^{6}$.

Le second numéro de Horizons pbilosopbiques consacré à la sémiotique, intitule, je le rappelle, "théories et champs d'applications", est ainsi nommé de manière fort opportune. En effet, ce qui est ici convoqué, c'est une jonction de lieux particuliers d'analyse dans un encadrement théorique. Trois exemples touchent de plus près la littérature: la question de son adaptation au cinéma, la notion de genre et la poéticité d'un texte. Si la première question est traitée par Alain Morency de manière plutôt classique, la seconde offre un territoire de réflexion peu fréquenté: Nycole Paquin aborde les genres, certes, mais dans le domaine des arts visuels (tableau d'histoire, portrait, paysage, nature morte, image abstraite et image d'écriture). Or, il pourra paraître fécond de mettre en regard la notion de genre littéraire: - Contrairement au genre littéraire qui détermine les horizons d'attente du lecteur, le genre pictural ne précède pas la perception des unités plastiques, il en découle." (p. 125) Enfin, Nathalie Watteyne livre une réflexion 
nourrie et féconde sur le "supplément poétique": "La manière de prendre parole nous indique comment un sujet ouvre une brèche entre son être (à délivrer) et le champ poétique (à accomplir). (p. 156 ${ }^{7}$

\section{Sociologie(s)/Histoire}

À l'Úniversité de Toronto se tenait en 1990 un colloque intitulé - Before 1860, Discourse/Language in Canada. Avant 1860, discours et langages au Canada . Sans doute devait-il revenir à Canadian Literature $^{8}$ de livrer une partie des communications prononcées à cette occasion. Une dizaine d'articles en anglais et en français abordent divers sujets liés aux XVII', XVIII e et XIX'e siècles: les Relations des jésuites, bien sû́r, mais aussi le discours des voyageurs, les canons littéraires au XIX $\mathrm{XX}^{\mathrm{e}}$ siècle, etc. Impossible ici de rendre compte de chacune des contributions et, de toute façon, le lecteur intéressé par la période ne pourra faire l'économie d'aucun article. Comme le signalent avec à propos Germaine Warkentin et Heather Murray dans l'Introduction, .The writing and assembling of these essays is [...] heuristic, rather than descriptive or prescriptive. ${ }^{9}$ (p. 13) Formulation élégante qui absout des manques, particulièrement celui des journaux (Gazette de Québec, Gazette de Montréal, etc.), véritables débuts, à bien des points de vue, d'une "littérature d'ici .

Certains articles ont, explicitement, un contenu et une problématique sociologique: je pense ici, dans le cadre des années 1940-1950, à cette querelle entre Robert Charbonneau et le Comité national des Écrivains français qu'étudie Élisabeth Nardout-Lafarge ${ }^{10}$. Sur à peu près la même époque, je tiens à signaler, cependant, une étude d'Yvan Cloutier sur "la circulation de deux produits, soit la psychanalyse et l'existentialisme, dans le champ canadien-français des années 1940-1960 et plus spécifiquement au rôle des dominicains pour Montréal et Ottawa ${ }^{11}$. Outré, comme dans le cas de l'article précédent, son incidence sur le domaine de l'histoire culturelle et l'histoire de l'édition, cette analyse pose un modèle de fonctionnement de la structure territoriale sur les processus d'importation/exportation et de légitimation des biens symboliques. Jusqu'à un certain point, psychanalyse et philosophie désignent les illustrations accidentelles d'une étude dont la logique d'un marché de biens symboliques peut, elle, être * exportée * pour scruter d'autres cas.

\section{Genres}

Assez peu d'études embrassent un genre tout entier, par la lunette d'une problématique particulière. Mais on sera sans doute intéressé, dans 
le cas du roman, par "Le roman des copines" d'Alexandra Jarque ${ }^{12}$. Il est impossible d'avoir lu quelques romans récents et n'avoir pas été frappé par une génération marquée par la non-territorialité ou l'errance, de même que par la dissolution des liens de sociabilité traditionnels ${ }^{13}$.

En ce qui a trait à la poésie, deux études substantielles composent à elles seules la partie critique du Courrier international d'études poétiques et se complètent mutuellement. Claude Filteau aborde la question de la modernité, dans la première moitié du XX' siècle, se fondant non pas sur la rupture de la thétorique traditionnelle comme indication, mais plutôt sur "cette ouverture de la pensée d̀ elle-même par un langage fondant le "vivre ensemble" ${ }^{14}$. Filteau lit ainsi les productions poétiques de l'École littéraire de Montréal jusqu'au manifeste de Paul-Émile Borduas. Quant à Claude Beausoleil, il he fait rien de moins qu'un parcours total, de Michel Bibaud aux "Figures de la révolte", de l'Ode au Québec, à la *Modernité et la Ville - de même qu'aux *Lieux expérimentaux *. Je me plais à reproduire cette petite phrase: *Urbaine et moderne, la poésie du Québec l'est depuis le début du siècle.. (p. 36) Et ces quelques lignes sur les trente dernières années:

La poésie des années 1970, avec ses expériences langagières poussées, s'était placée dans une position illusoire: la page se donnait comme univers habitable. Les années 1980 ont été celles de la recherche intérieure. Dès le début de la décennie 1990, les choses ont changé et la poésie a manifesté le désir de pactiser avec son public.. (p. 45)

La littérature de jeunesse attire de plus en plus l'attention des chercheurs, résultat, sans doute, de l'importance qu'elle a prise depuis quelques années. Dans sa présentation des deux numéros de Présence francopbone qu'elle a préparés sur le sujet, Suzanne Pouliot ne manque pas de noter la légitimation accrue dont la littérature de jeunesse a fait l'objet. Fidèle à son mandat, la revue livre une dizaine d'articles de toutes provenances, dont trois se penchent sur des cas québécois. Après avoir.brossé un tableau des deux dernières décennies, Manon Poulin aborde les cas des maisons d'édition Ovale et La Courte Échelle. Daniel Mativat scrute la situation ambiguë du statut de l'auteur qui œuvre dans le domaine de la littérature de jeunesse: il s'agit d'une réflexion bien documentée et fort à propos. Enfin, Sylvie Faure étudie le volet de la littérature de jeunesse aux Éditions Leméac, portant attention au catalogue et aux créneaux exploités dans le cadre général de l'évolution de Leméac jusqu'à sa faillite en $1988^{16}$.

Un mot sur cet aspect pourtant fondamental de la création, en particulier des ateliers: s'écartant de son habitude, la Revue des Forges offre quelques articles où des professeurs qui ont animé des ateliers de création réfléchissent sur cette pratique. La plupart des intervenants, 
Bruno Roy, René Lapierre et Joseph Bonenfant entre autres - et sans concertation j'imagine - ont senti le besoin de positionner (comme on dit de nos jours) l'écriture littéraire par rapport à l'écriture technique, le sujet par rapport au code, la création vis-à-vis de l'institution. Ainsi, à qui lui demande: "Que voulez-vous au justè? ou, traduction simultanée, quels sont les impératifs institutionnels de l'écriture?", René Lapierre rétorque - que là première tâche est là : cesser de croire en ce domaine que quelqu'un peut vouloir à leur place, parler ou écrire en leur nom. Faire le vide, laisser parler la petite voix qui vient du fond d'eux-mêmes - appelons-la la voix de l'âme. ${ }^{17}$. C'est ce qui distingue l'écriture qui épelle le moi au lieu du code.

\section{Auteurs}

Pour terminer ce survol, je voudrais signaler quelques études sur des auteurs particuliers. Jacques Ferron d'abord, auquel Études littéraires a consacré un numéro tout entier ${ }^{18}$. Une autre revue accorde aussi deux études à Ferron: les Cabiers francopbones d'Europe CentreOrientale, dont il s'agit du premier numéro. Cette "revue annuelle de pluriculturalisme, ne veut pas donner dans la mièvrerie du melting-pot ou dans la dictature des parties sur le tout: son avant-propos caustique par Arpad Vigh nous fait comprendre que le pluriculturalisme ne cherche pas à imiter certains modèles canadiens * tendant à noyer l'autonomisme québécois dans une diversité folklorique parfaitement compatible avec une normalité anglophone ${ }^{19}$.. On y trouve donc des études de Vigh et de Reinhart Hosch sur Ferron - ce dernier y reprenant le texte paru dans Études littératres. Notons en outre une étude intertextuelle de L'Hiver de force de Ducharme (Eszter Podoski) et, bien que trop brève, une réflexion sur le "Bilinguisme littéraire au Canada ${ }^{20}$. (Katalin Kurtosi). Enfin, dans le domaine de la poésie, signalons que l'étude d'écRiturEs de Paul-Marie Lapointe, par André Marquis dans Canadian Literature, s'apparente fort bien aux propos tenus dans la Revue des Forges sur les ateliers de création, du moins en conclusion ${ }^{21}$.

Je termine en contredisant avec joie (et partiellement, bien sûr!) mon point de départ: la poésie, au moins, se donne une bibliographie annuelle, pour une deuxième année consécutive: La Poésie au Québec (Revue critique) 1990. L'on y trouve la recension critique des œuvres de quelque 150 auteurs, deux documents et un *Palmarès ${ }^{22}$. Puisse l'initiative se poursuivre et susciter une saine émulation!

1. Horizons pbilosopbiques, eSémiotiques I': mises au point, mises en question., vol. $1, \mathrm{n}^{\circ} 1$ (automne 1990) et -Sémiotiques 2: théories et champs d'application:, vol. I, $\mathrm{n}^{\circ} 2$ (printemps 1991). 
2. Pour des considérations analogues, voir Lucie Robert, -Le fétichisme de la littérature., dans L'Histoire littéraire. Tbéories-mábodes-pratiques, sous la direction de Clément Moisan, Québec, P.U.L., 1989, p. 17-24.

3. - Esquisse d'une theorie de la forme dramatique ., Langages, $n^{\circ} 12,1967$, p. 71-93.

4. Le théâtre québécois: stimulation ou communication?, Voix \& Images, vol. I, $\mathrm{n}^{\circ} 2$ (1975), p. 220-240.

5. Â cette fin, on pourra d'ailleurs aller dans le detail de la plupart de ces questions avec, de Louise Vigeant également, La Lecture du spectacle théäral, Laval, Mondia, 1989, 228 p. Notons enfin que ce numéro de Horizons pbilosopbiques n'est pas en reste sur la psychanalyse avec un article de Simon Harel, a L'ecriture réparatrice.

6. Louise Vigeant, .De la métonymie $i \mathrm{la}$ métaphore: h didascalie comme piste, Jeu, Scénographie , n॰ 62, mars 1992, p. 33-40.

7. Ces trois articles sont d'Alain Morency, - L'adaptation de la littérature au cinéma ., p. 103-123; de Nycole Paquin, -Sémiotique des "genres". Une theorie de la réception, p. 125-135; de Nathalie Watteyne, - Le supplément poétique,, p. 137-160. Je signale enfin, en plus d'articles portant sur la sémiotique comme théorie et praxis par Gilles Therien, Jean-Guy Meunier et Nadia Khouri, des études sur le corps et ses langages (Jean-Jacques Courtine), l'ouvre picturale (Marie Carani) et la musique, en l'occurrence Glen Gould et les Variations Goldberg de Bach.

8. Canadian Literature, -Discours in Early Canada,$n^{\circ} 130$, automne 1991.

9. Traduction: -L'écriture et la reunion de ces articles relevent d'une approche davantage $[. .$.$] heuristique que descriptive ou prescriptive. .$

10. - Robert Charbonneau et le C.N.E.: les enjeux de la polémique, Rerue francopbone de Louisiane, vol. V, n' 1, printemps 1990, p. 71-78.

11. Les dominicains et l'import-export: psychanalyse et existentialisme au Québec., Horizons pbilosopbiques, vol. II, n 1, automne 1991, p. 92.

12. Possibles, Générations 91 , vol. XV, $n^{\circ} 2$, printemps 1991, p. 31-42.

13. Voir par exemple Gilles Marcotte, Générations ", dans L'Âge de la prose, sous la direction de Lise Gauvin et Franca Marcato-Palzoni, Montréal/Rome, VLB éditeur/Bulzoni, 1992, p. 19-27.

14. Claude Filteau, -La question de la modernité 1895-1948, - Le Courrier du Centre international d'études poétiques, \& Poésies québécoises , n 190, avril-juin 1991, p. 8.

15. Claude Beausoleil, - Poésie québécoise et modernité ou Les passions de l'identité •, ibid., p. 27-46.

16. Manon Poulin, : L'édition québécoise pour la jeunesse se porte bien, Présence francopbone, -la littérature de jeunesse-I -, $n^{\circ} 38,1991$, p. 33-51; Daniel Mativat, - Littérature de jeunesse au Québec: marche du livre et statut socio-economique des ecrivains,, ibid., p. 85-94; Sylvie Paure, - De la joie de lire aux jours de fête: la littérature de jeunesse aux éditions Leméac ', Présence francopbone, $n^{\circ} 39,1991$, p. 31-44. Je voudrais signaler par la même occasion, dans ce numéro mais horsthème, a Idéologies et écritures des femmes au Québec (1970-1980). par Bénédicte Mauguière, p. 111-125.

17. René Lapierre, :Ateliers de création et attente institutionnelle, Reurue des Fonges, $\mathrm{n}^{\circ} 32$, octobre 1991, p. 19.

18. Etudes littéraires, -Jacques Ferron en exotopie., vol. XXIII, n` 3, hiver 1990-1991.

19. Cabiers francopbones d'Europe Centre-Orientale, $n^{\circ} 1,1991, \mathrm{p} .6$.

20. Il s'agit la d'une question importante à propos de laquelle beaucoup reste à faire: voir par exemple Ben-Z. Shek, Frencb-Canadian \& Québécois Novels, . The Problems of Translation,, Toronto, Oxford University Press, 1991, p. 136-144.

21. André Marquis, -Démystification ou fumisterie?: à propos d'écRiturEs de PaulMarie Lapointe, Canadian Literature, - Writing \& Cultural Values,, n* 130, automne 1991, p. 118-128.

22. La Pósie au Québec (Revue critique), 1990, Écrits des Forges/Cégep Joliette-De Lanaudière, 1991, $213 \mathrm{p}$ 